\title{
Non-linear Local Registration of Functional Data
}

\author{
Isabelle Corouge $^{1}$, Christian Barillot ${ }^{1}$, Pierre Hellier ${ }^{1}$, Pierre Toulouse $^{2}$, and \\ Bernard Gibaud ${ }^{2}$ \\ 1 IRISA, INRIA-CNRS, Campus de Beaulieu, 35042 Rennes Cx, France \\ \{icorouge, cbarillo, phellier\}@irisa.fr \\ http://www.irisa.fr/vista \\ 2 IDM laboratory, Faculty of Medicine, University of Rennes I, 35043 Rennes Cx, \\ France \\ \{Pierre.Toulouse, Bernard.Gibaud\}@univ-rennes1.fr
}

\begin{abstract}
Within the scope of three-dimensional brain imaging we propose an inter-individual fusion scheme to register functional activations relatively to anatomical cortical structures, the sulci. This approach is local and non-linear. It relies on a statistical sulci shape model accounting for the inter-individual variability of a population of subjects, and providing deformation modes relatively to a reference shape (a mean sulcus). The deformation field obtained between a given sulcus and the reference sulcus is extended to a neighborhood of the given sulcus by using the thin-plate spline interpolation. It is then applied to the functional activations associated with this sulcus. This approach is compared with other classical matching methods.
\end{abstract}

\section{Introduction}

In the context of inter-individual normalization, we address in this paper the registration of $3 \mathrm{D}$ anatomical and functional data, i.e. data from various subjects and/or acquired according to various modalities (e.g. magnetic resonance imaging (MRI) for anatomical data, magnetoencephalography (MEG) or functional magnetic resonance imaging (fMRI) for functional data). We intend to grasp the high inter-individual variability implied by such data with a shape model. Deformable models are a powerful tool to image analysis [1]. Some of them use modal analysis techniques lying on a physical approach [2], 3] or on a statistical approach 4, 5]. In this kind of model, adequation between model and data is improved by introducing prior knowledge thanks to a training set. These models are able not only to represent the shape of an object but also the way it can vary. They are generally used for segmentation purpose. Within the framework of anatomo-functional normalization, it is interesting to use the modeling of deformations to register scattered data associated with modeled structures of interest. Thus to register functional activations we rely on the modeling of anatomical structures, the sulci, which are relevant landmarks for such purpose; the registration being finally achieved thanks to a technique based on the thinplate spline interpolation [6], [7]. 
In Sect. 2]we describe the construction of the statistical model of cortical sulci by learning a set of shapes. The training stage is first detailed, then we present the statistical analysis we use, i.e the principal component analysis (PCA). In Sect. 3. we present the thin-plate spline method and its use combined to the model exploitation in the local and non-linear registration of functional activations, MEG dipoles. We compare this approach with a local rigid approach and with global methods (rigid and non-rigid).

\section{Statistical Model of Cortical Sulci}

\subsection{Training}

Cortical sulci are anatomical structures whose shape is complex. We dispose of a parametric representation of these shapes of interest [8] describing them by their median surface. This one is extracted from MRI volumes by the "active ribbon" method and is eventually modeled by a cubic B-spline surface, which is well adapted to model free form objects. The spline, parameterized by $u$ and $v$, is described by $n b p$ knots and $n b c=n b c_{-} u * n b c_{-} v$ control points where $n b c_{-} u$ (resp. $\left.n b c_{-} v\right)$ is the number of control points in the direction associated with parameter $u$ (resp. $v$ ). In the case of sulci, the parametric direction $u$ represents the length of the sulcus and the direction $v$ its depth. Giving $n b c$ control points completely defines the sulcal surface. Consequently, we can represent a sulcus by the vector of its knots or its control points. The ratio $n b c / n b p$ defines the smoothing factor: the smaller this ratio, the smoother the surface (we have chosen $n b c / n b p=1 / 24$ ). The main advantages to use control points are their lower number and their complete representation of each surface.

The statistical technique used here needs to establish the point to point correspondences between all shapes of the training set. This implies a resampling stage so that the sulci have the same number of points and a registration stage in order to express them in the same system of reference. Each sulcus is initially expressed in its image reference system which is different from one patient to another. The idea is to associate its own system of reference with each sulcus, built so that it is common to all sulci. We call it "local system of reference". It is then just needed to determine the rigid transformation (rotation+translation) aligning all local systems of axes and to apply it to the associated shapes.

Let $\mathcal{R}_{s}\left(O_{s}, \mathbf{u}_{\mathbf{s}}, \mathbf{v}_{\mathbf{s}}, \mathbf{w}_{\mathbf{s}}\right)$ be the system of reference local to the sulcus. The axes $\mathbf{u}_{\mathbf{s}}, \mathbf{v}_{\mathbf{s}}$ and $\mathbf{w}_{\mathbf{s}}$ are defined as the axes of inertia of the sulcal surface, and are decided to be so that $\mathbf{u}_{\mathbf{s}}$ follows the length of the sulcus, $\mathbf{v}_{\mathbf{s}}$ its depth and $\mathbf{w}_{\mathbf{s}}$ its normal. This discrimination between the 3 axes is first carried out by considering that $\mathbf{u}_{\mathbf{s}}$ (resp. $\mathbf{v}_{\mathbf{s}}$ ) is the axis of inertia the "most collinear" with the $n b c_{-} u$ (resp. $n b c_{-} v$ ) pseudo-parallel directions; each of them being defined by the two extremities of a sulcus' line in direction $u$ (resp. $v$ ). Then $\mathbf{w}_{\mathbf{s}}$ is obtained by vector product: $\mathbf{w}_{\mathbf{s}}=\left(\mathbf{u}_{\mathbf{s}} \wedge \mathbf{v}_{\mathbf{s}}\right)$. At last the origin $O_{s}$ is the center of mass of the sulcus.

The sulci have now to be expressed in their local systems of reference. It amounts to determining, for each sulcus, the matrix $\mathbf{M}$ defining the change of 
basis from the local system of reference $\mathcal{R}_{s}$ towards the image system of reference, let it be $\mathcal{R}(O, \mathbf{u}, \mathbf{v}, \mathbf{w})$. Let $\mathbf{R}$ and $\mathbf{t}$ be the rotation matrix and the translation vector of the inverse change of basis $\mathbf{M}^{-1}$ (i.e. from $\mathcal{R}$ towards $\mathcal{R}_{s}$ ). Then in homogeneous coordinates:

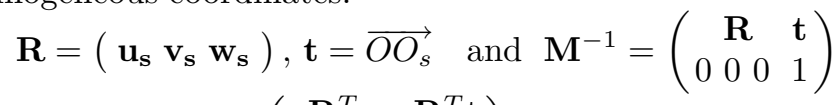

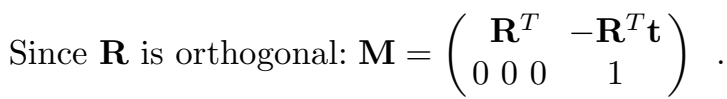

Applying this rigid transformation to all the points of each sulcus aligns the training set as illustrated on Fig. 11a. Since the image data are acquired to the same scale, we decided not to perform any homothety. First, it enables to avoid one more transformation, then the inter-individual size variation will thus be grasped by the shape model.

The next stage consists in resampling the sulci of the training set. We resample the elements of the training population on the one which has the most sample points, spline properties ensuring that the original shapes are preserved. Once the sulci are resampled and aligned, the matching is performed by just assigning control point to control point according to their curvilinear abscissa.
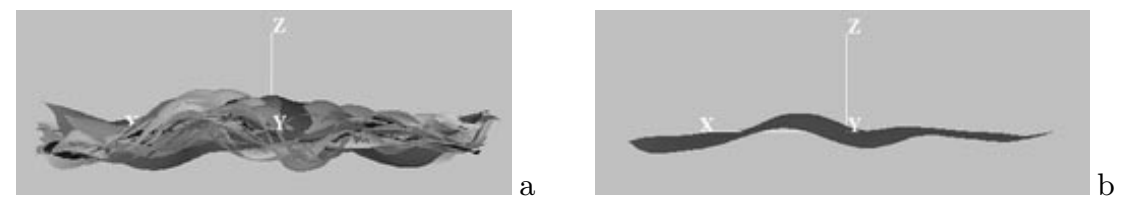

Fig. 1. a) A side view of a database of 18 left central sulci aligned in the local system of reference. b) A side view of the mean left central sulcus of this database.

\subsection{Statistical Analysis of Deformations}

The statistical analysis of the training set leads to a modeling of cortical sulci and of their variations. The model captures the shape variability observed within the training set. Indeed, the statistical analysis reveals the main modes of variation relative to a prototype shape, representative of the considered class. We use a principal component analysis which enables to represent data in a new basis, orthogonal, and which suppresses the redundancy of information. Moreover, this analysis enables a modal approximation.

Principal Component Analysis. Let $\mathcal{P}$ be the training population made up of $N$ elements, $\mathbf{x}_{i} \in \mathcal{P}$ a shape, $\overline{\mathbf{x}}$ the mean shape on $\mathcal{P}, \mathbf{C}$ the covariance matrix. A shape $\mathbf{x}_{i}$ is represented by the vector of control points of the spline which models the median surface of the sulcus:

$$
\mathbf{x}_{i}=\left(x_{i_{1}}, y_{i_{1}}, z_{i_{1}}, \ldots, x_{i_{n}}, y_{i_{n}}, z_{i_{n}}\right)^{T} \text { with } n=n b c .
$$

The mean shape, representative of the studied class, and the covariance matrix are given by: 


$$
\overline{\mathbf{x}}=\frac{1}{N} \sum_{i=1}^{N} \mathbf{x}_{i} \text { and } \mathbf{C}=\frac{1}{N} \sum_{i=1}^{N} d \mathbf{x}_{i} d \mathbf{x}_{i}^{T} \text { with } d \mathbf{x}_{i}=\mathbf{x}_{i}-\overline{\mathbf{x}}
$$

Diagonalizing the covariance matrix $\mathbf{C}$ provides the new modal basis $\boldsymbol{\Phi}$ :

$$
\mathbf{C}=\boldsymbol{\Phi} \boldsymbol{\Lambda} \boldsymbol{\Phi}^{T}, \Lambda=\operatorname{diag}\left(\lambda_{1}, \ldots, \lambda_{3 n}\right) \text { with } \lambda_{1} \geq \lambda_{2} \geq \ldots \geq \lambda_{3 n} .
$$

Then any shape $\mathbf{x}$ can be written: $\mathbf{x}=\overline{\mathbf{x}}+\mathbf{\Phi} \mathbf{b}$ where $\mathbf{b}=\left(b_{1}, \ldots, b_{3 n}\right)^{T}$ is the vector of modal amplitudes of deformation and $(-\boldsymbol{\Phi} \mathbf{b})$ corresponds to the deformation vectors in each point of $\mathbf{x}$ towards the mean shape. Since the eigenvalue $\lambda_{i}$ is the variance explained by the $i^{t h}$ mode, a large part of the variability can be explained by retaining only the first $m$ modes. The value $m$ is chosen so that $\sum_{i=1}^{m} \lambda_{i}$, the variance explained by the first $m$ modes, represents a proportion, sufficiently important of the whole variance: $\lambda_{T}=\sum_{i=1}^{3 n} \lambda_{i}$. Retaining only $m$ modes enables us to achieve a modal approximation:

$$
\left\{\begin{array}{l}
\mathbf{x}=\overline{\mathbf{x}}+\boldsymbol{\Phi}_{\mathbf{m}} \mathbf{b}_{\mathbf{m}} \\
\mathbf{b}_{\mathbf{m}}=\boldsymbol{\Phi}_{\mathbf{m}}{ }^{T}(\mathbf{x}-\overline{\mathbf{x}})
\end{array}\right.
$$

where $\boldsymbol{\Phi}_{\mathbf{m}}$ is a submatrix of $\boldsymbol{\Phi}$ containing the first $m$ eigenvectors of $\mathbf{C}$, thus defining the modal approximation basis. The vector $\mathbf{b}_{\mathbf{m}}=\left(b_{1}, \ldots, b_{m}\right)^{T}$ represents a shape in the $m$-dimensional space defined by the principal components. This space is interesting since it is of lower dimension $(\operatorname{dim} m)$. However, $\mathbf{b}_{\mathbf{m}}$ must be constrained in order to represent an "allowable" shape (i.e. a shape consistent with the learnt shapes). Given the assumption that the distribution of vectors $\mathbf{x}_{\mathbf{i}}$ is normally distributed (i.e. gaussian distribution), the range of variability of each $b_{i}$ is typically such as: $-3 \sqrt{\lambda_{i}} \leq b_{i} \leq+3 \sqrt{\lambda_{i}}$.

Results. Several tests have been carried out by making the cardinal of the training population vary (up to 85 sulci) and also by changing the type of sulci (central right and left sulcus, lateral sulcus, superior frontal sulcus, ....). We present the results obtained on a training set made up of the 18 left central sulci registered in the previous stage. Figure 2 shows the predominance of the first modes. Indeed, the first 5 modes explain a large part of the total variation (about $70 \%$ ). The first mode explains on its own almost $30 \%$ of the total variation (whereas a sulcus is described by 104 control points, that is to say 312

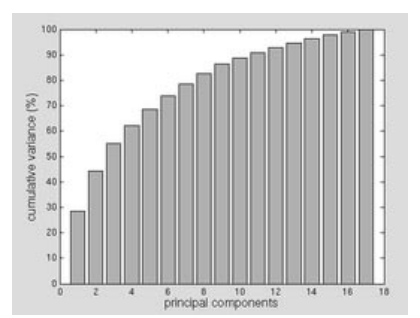

Fig. 2. Cumulative variance according to the number of principal components retained. 

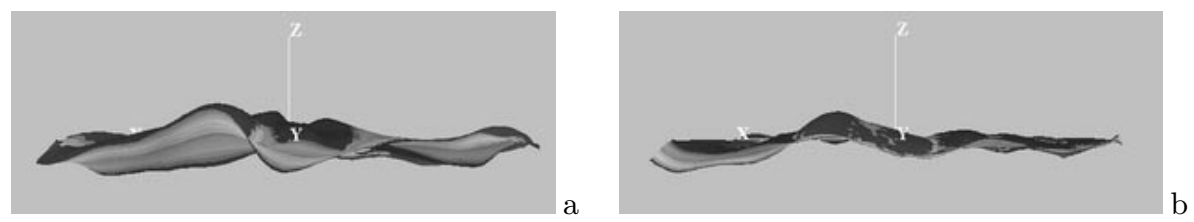

Fig. 3. a) Variations of the first mode around the mean sulcus, $-3 \sqrt{\lambda_{1}} \leq b_{1} \leq$ $+3 \sqrt{\lambda_{1}}$. b) Variations of the $15^{\text {th }}$ mode around the mean sulcus, $-3 \sqrt{\lambda_{15}} \leq b_{15} \leq$ $+3 \sqrt{\lambda_{15}}$.

variables, and by more than 8000 variables if knots are considered). The statistical modeling seems to be appropriate to express the shapes and the variations in a compact way. Figure 3 a shows the variations due to the first mode. They are mainly relative to the length and to the torsion of the sulcus. On the contrary, Fig. 3b illustrates the minor influence of the $15^{\text {th }}$ mode: the deformations are hardly distinct, all the sulci are almost superimposed to the mean shape.

\section{Deformation Fields and Non-linear Registration}

The deformation field $\left(-\mathbf{\Phi}_{\mathbf{m}} \mathbf{b}_{\mathbf{m}}\right)$ obtained between a given sulcus and the reference sulcus (the mean sulcus in our case) can be extended to a local neighborhood of the considered sulcus by using the thin-plate spline interpolation [6]. It can then be applied to any object associated with this sulcus. We take advantage of this extension of the deformation field $\left(-\mathbf{\Phi}_{\mathbf{m}} \mathbf{b}_{\mathbf{m}}\right)$ to register scattered data located in the left central sulcus area towards a mean space.

\subsection{The Thin-Plate Spline Method}

The use of thin-plate spline interpolation for registration purpose in medical imaging was first proposed by Bookstein. In [6], he proposes an algebraic approach to describe deformations specified by two sets of corresponding points. This method provides an interpolation function $f$ which maps one of the two sets of corresponding points, the source set, onto the other one, the target set. Moreover, the function $f$ is defined in some neighborhood of the set of source points so that it can be applied to a point in the source space to find its homologous in the target space.

Let $\mathcal{P}=\left\{P_{i}\left(x_{i}, y_{i}, z_{i}\right), i=1, \ldots, n\right\}$ be the set of source points in the Euclidean space, and $\mathcal{V}=\left\{V_{i}=\left(x_{i}^{\prime}, y_{i}^{\prime}, z_{i}^{\prime}\right), i=1, \ldots, n\right\}$ the set of target points. The set $\mathcal{P}$ describes a shape $\mathbf{x}$, expressed by $\overline{\mathbf{x}}+\boldsymbol{\Phi}_{\mathbf{m}} \mathbf{b}_{\mathbf{m}}$ according to our model. Let $r_{i j}=\left|P_{i}-P_{j}\right|$ be the Euclidean distance between two source points $P_{i}$ and $P_{j}$. Then the function $f$ is the sum of two terms: an affine part which represents its behavior at infinity, and a second part which is asymptotically flat:

$$
f(x, y, z)=a_{1}+a_{x} x+a_{y} y+a_{z} z+\sum_{j=1}^{n} w_{j} U\left(\left|P_{j}-(x, y, z)\right|\right)
$$


where

- the basis function $U$ is the fundamental solution of the biharmonic equation $\Delta^{2} U=\delta(0,0), \delta$ being the Kronecker's function. It can be shown 7 that the equation of a thin uniform metal plate originally flat and now bent by vertical displacements is directly related to the biharmonic equation. In $3 \mathrm{D}$ the function $U$ is $U(r)=|r|$;

- the coefficients $\mathbf{a}=\left(a_{1}, a_{x}, a_{y}, a_{z}\right)^{T}$ and $\mathbf{w}=\left(w_{1}, w_{2}, \ldots, w_{n}\right)^{T}$ are obtained by solving the linear system:

$$
\left\{\begin{array}{l}
\mathbf{K w}+\mathbf{P a}=\mathbf{v} \\
\mathbf{P}^{T} \mathbf{w}=0
\end{array} \quad \text { where } \quad \mathbf{P}=\left(\begin{array}{cccc}
1 & x_{1} & y_{1} & z_{1} \\
\vdots & \vdots & \vdots & \vdots \\
1 & x_{n} & y_{n} & z_{n}
\end{array}\right),\right.
$$

$\mathbf{K}$ is a $n \times n$ matrix having the general term $\left(U\left(r_{i j}\right)\right)$, and $\mathbf{v}$ is the vector of one coordinate of the target set (e.g. $\mathbf{v}=\left(x_{1}^{\prime}, \ldots, x_{n}^{\prime}\right)$, what implies that (1) must be declined for $\left.f_{x}(x, y, z), f_{y}(x, y, z), f_{z}(x, y, z)\right)$.

Regarding the target set as the mean shape $\overline{\mathbf{x}}$, the deformation field $\left(-\mathbf{\Phi}_{\mathbf{m}} \mathbf{b}_{\mathbf{m}}\right)$ is then represented by the elements of $(\mathbf{w} \mid \mathbf{a})$, and it is extended outside the source shape $\mathbf{x}$ thanks to the function $f$.

\subsection{Results}

The statistical modeling of anatomical structures such as cortical sulci can now be used to register functional activations in a non-linear and local way by using the method described above. We first detail the results obtained through this method (NLL), and second we compare this approach with the following methods:

- a global rigid method (GR): the registration by maximization of mutual information [9], 10];

- a global piecewise affine registration (PS): the Talairach Proportional Squaring 11 ;

- a non-linear global registration (NLG): a method based on optical flow and a robust optimization scheme [12];

- a local rigid method (LR): the local registration realized through the transformation described by the matrix $\mathbf{M}$ (see Sect. 2.1). This method will be evoked over all this part since it is a constant in all methods.

For all methods, the functional data to register are MEG dipoles corresponding to a somatosensory activation of right hand fingers (thumb, index, little finger) performed for 15 subjects of the 18 subjects of our database (see Sect. 21). MEG current dipoles have been reconstructed using a spatiotemporal algorithm [13] and selected by choosing the most significant one in the $45+/-15 \mathrm{~ms}$ window. These functional activations are located in the central sulcus area. 

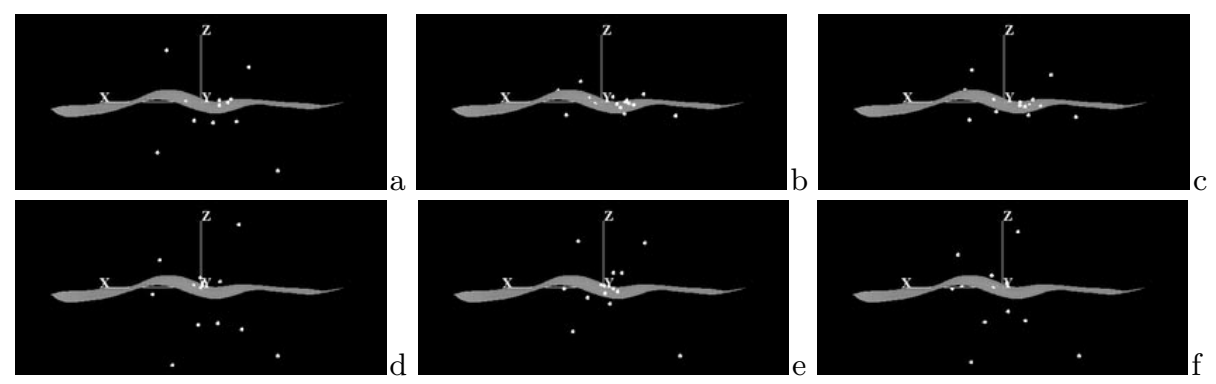

Fig. 4. Registration of MEG dipoles (somatosensory activation of the thumb): the sulcus is the mean left central sulcus. a) Method LR: the dipoles are rigidly registered. b), c) Method NLL: the dipoles are registered via the deformation field , b) $m=17$, c) $m=5$. d) Method GR. e) Method PS. f) Method NLG.

Method NLL. We apply the thin-plate spline method to merge anatomical and functional information in the central sulcus mean space. First, we rigidly register each dipole towards the local space by applying the transformation described by the corresponding matrix $\mathbf{M}$ (see Sect. 2), i.e. by using the LR method. Then, for each subject, we compute the "field" (w $\mid \mathbf{a})$ between the left central sulcus of this subject and the mean sulcus. We apply it to the 3 dipoles of this subject, the field $\left(-\mathbf{\Phi}_{\mathbf{m}} \mathbf{b}_{\mathbf{m}}\right)$ being computed with all the modes $(m=17)$. Figure $4 \mathbf{b}$ shows that dipoles gather around the plane of the mean sulcus. Moreover, the covariance along $x, y$ and particularly $z$ is considerably reduced (see Table 1 , first row). We present a second test in which we consider only 5 modes in the construction of $\left(-\mathbf{\Phi}_{\mathbf{m}} \mathbf{b}_{\mathbf{m}}\right)$. This approximation smoothes the sulcus and discards minor modes possibly resulting from potential segmentation errors of initial data. Results are presented Fig. 4c and Table 1. The gathering towards the mean plane and the decrease of the covariance are less than the ones of the previous test, but still significant.

Table 1. The covariance along $x, y$ and $z$ of MEG dipoles for somatosensory activations (thumb, index, little finger) after local rigid registration (LR), thinplate splines interpolation based registration (NLL) for $m=17$ and $m=5$, and global registration (methods GR, PS, NLG).

\begin{tabular}{|c|c|c|c|}
\hline & $\overline{\mathrm{LR}}$ & $\operatorname{NLL}(m=17)$ & NLL $(m=5)$ \\
\hline thumb & 90.825 .4169 .99 & $77.3321 .98 \quad 7.3$ & 80.8820 .3916 .67 \\
\hline index finger & 90.8931 .5871 .85 & 85.4626 .255 .96 & 82.3926 .9911 .81 \\
\hline \multirow[t]{2}{*}{ little finger } & $97.7933 .36 \quad 81.94$ & 90.1834 .268 .05 & 88.2932 .9921 .91 \\
\hline & $\overline{G R}$ & $\overline{\mathrm{PS}}$ & $\overline{\mathrm{NLG}}$ \\
\hline thumb & 92.0122 .93111 .18 & $\begin{array}{lllll}76.67 & 16.98 & 69.21\end{array}$ & 81.5625 .3798 .11 \\
\hline index finger & 94.1327 .7597 .98 & 83.3125 .2669 .41 & 86.5238 .5190 .01 \\
\hline little finger & 99.3928 .68111 .31 & 89.3622 .9362 .83 & 91.6939 .31102 .99 \\
\hline
\end{tabular}


Comparison. We apply each of the 3 global methods to MEG data; then, for comparison purpose, we place the resulting dipoles in the local space via the LR method. Figure 4 shows that method NLL gathers the dipoles around the reference mean sulcus more than the other ones. This visual result is confirmed by computing the dipole localization covariance along each axis (see Table1). Global methods, linear or not, lead to quite similar dispersion. However method PS leads to inferior covariances than method GR and NLG. This may be explained since method PS is by construction relevant and precise in the central sulcus area. To light the significant difference observed between global methods and method NLL we may note that global methods GR, PS and NLG rely more on luminance information than on anatomical information, whereas the NLL method is fully based on anatomical constraints. That point may be a motivation to introduce local anatomical contraints in global registration process [14], 15].

\section{Conclusion}

We have presented a statistical model of cortical sulci built by performing a modal analysis (PCA) on a training population. This model accounts for the deformations of the sulci between individuals, and the achieved tests show the relevance of the obtained deformation modes. This modeling has the advantage to lie in a "mean space". The sulci thus modeled can be used as landmarks in the registration of MEG dipoles towards the mean space. This registration is carried out via the thin-plate spline method. Results show a significant difference between this local and non-linear method and the global methods we presented. Interpretation remains delicate all the more so as no ground truth is available. Finally, the registration framework presented here is general and not restricted to MEG activities.

\section{References}

1. McInerney, T., Terzopoulos, D.: Deformable Models in Medical Image Analysis: A Survey. Medical Image Analysis, 1(2):91-108 (1996).

2. Subsol, G., Thirion, J.P., Ayache, N.: A General Scheme for Automatically Building 3D Morphometric Anatomical Atlases: Application to a Skull Atlas. MedIA, 2(1):37-60 (1998).

3. Martin, J., Pentland, A., Sclaroff, S., Kikinis, R.: Characterization of Neuropathological Shape Deformations. IEEE Trans. on PAMI, 20(2):97-112 (1998).

4. Kervrann, C., Heitz, F.: A Hierarchical Statistical Framework for the Segmentation of Deformable Objects in Image Sequences. IEEE CVPR, 724-728 (1994).

5. Cootes, T.F., Taylor, C.J., Cooper, D.H., Graham, J.: Active Shape Models - their training and application. CVIU, 61(1):38-59 (1995).

6. Bookstein, F.: Principal Warps: Thin-plate splines and the decomposition of deformations. IEEE Trans. on PAMI, 11(6):567-585 (1989).

7. Bookstein, F.L., Green, D.K.: A Feature Space for Derivatives of Deformations. IPMI, LNCS, 687:1-16 (1993). 
8. Le Goualher, G., Barillot, C.,Bizais, Y.: Modeling Cortical Sulci with Active Ribbons. IJPRAI, 8(11):1295-1315 (1997).

9. Collignon, A., Vandermeulen, D., Suetens, P., Marchal, G.: 3D Multi-Modality Medical Image Registration using Feature Space Clustering. CVRMed, 195-204 (1995).

10. Viola, P., Wells, W.: Alignment by Maximization of Mutual Information. Proc. Int. Conf. Computer Vision, 15-23 (1995).

11. Talairach, J., Tournoux, P.: Co-planar Stereotaxic Atlas of the Human Brain. Georg Thieme Verlag, Stuttgart (1988).

12. Hellier, P., Barillot, C., Mémin, E., Pérez, P.: An energy-based framework for dense 3D registration of volumetric brain image. IEEE CVPR, 2:270-275 (2000).

13. Schwartz, D., Badier, J.M., Bihoué, P., Bouliou, A.: Evaluation with realistic sources of a new MEG-EEG spatio-temporal localization approach. Brain Topography, 11(4):279-289 (1999).

14. Collins, L., Le Goualher, G., Venugopal, R., Caramanos, A., Evans, A., Barillot, C.: Cortical Constraints for Non-linear Cortical Registration. VBC, LNCS, 1131:307316 (1996).

15. Hellier, P., Barillot, C.: Cooperation Between Local and Global Approaches to Register Brain Images. IPMI, LNCS, 2082:315-328 (2001). 